\title{
Participatory Human-Centered Design: User Involvement and Design Cross-Fertilization
}

\author{
Guy A. Boy and Nadja Riedel \\ ${ }^{1}$ Florida Institute for Human and Machine Cognition (IHMC) \\ 40 South Alcaniz Street, FL 32502 Pensacola, USA \\ ${ }^{2}$ d-Lido design + development, 5. Quergässchen 2, 86152 Augsburg, Germany \\ gboy@ihmc.us, nrad-ligo.com
}

\begin{abstract}
Design and development of new instruments requires much attention with respect to safety, performance and comfort. Introducing new technology is a matter of taking care of past user experience on current technology and anticipating possible user experience on prototypes incrementally developed. The intricate spiral combination of prototyping and formative evaluations provides excellent support to include end-users in the design and development process. Human-centered design is also a combination of both analytical and usercentered (experimental) approaches. We cannot get rid of analyzing humanmachine interaction using methods such as GOMS for example, and neither using professional design expertise. These methods provide an envelope of usability and usefulness issues; some are directly applicable, others issues require an experimental user-centered evaluation, i.e., real professional users are needed. Usability engineering is now very much used in industry and provides good results. Crucial problems are not technical any longer; they are financial, legal, social and finally relational. The various actors who will have an influence on the product being developed should participate. Participatory design enables to improve awareness of product attributes, i.e., what the product is really for, and how it should be made and used. A running example of the design of a new flight attendant panel to be included in the cabin of commercial aircraft is presented to support methodological claims and demonstrate approach soundness.
\end{abstract}

Keywords: Human factors, HCI design, user involvement.

\section{Introduction}

During the 1990's, practice in cognitive engineering and human-computer interaction mainly focused on usability engineering [6] where we, i.e., members of the HCI community, tried to assess user interfaces of interactive systems and develop appropriate usability testing techniques. We wanted user interfaces to be easy to learn, efficient and pleasurable to use. We also wanted that users be able to easily recover from errors, or anticipate and avoid big risks as much as possible. It seems that this is routine practice in industry. However, even if there are procedures and rules that 
support these human-centered design efforts, it is mandatory to focus more on user requirements and on the integration of potential users in a real participatory way. In addition, a good design is not something that is created in a day by a single individual, but by a team of experts incrementally modifying prototypes toward a mature product. This part seems to be difficult and not fully mastered yet. Main reasons come from legal, financial, commercial, cultural, organizational and social issues. In this paper, we will not develop legal issues. Financial issues mainly deal with the cost/benefit issues of human factors integration in design activities [5]. Commercial and cultural issues are obviously related because participatory human-centered design (PHCD) cannot be carried out without cooperation between designers, manufacturers and clients. This paper will focus on the later issues where social relations are keys for the success of PHCD. PHCD still needs to incorporate these issues in a rational and systematic way because it remains a difficult and risky exercise. In this paper, we present the results of a study that was carried out over the last two years on the design of a novel interface to onboard cabin systems manipulated by flight attendants. The main goal was to harmonize the way users interact with this interface. Indeed, harmonization has become a key issue in environments that are populated by several interaction styles coming from various suppliers. The need for integration is a real central issue. Again, it is not a purely technical issue, but a social and economical one.

\section{User Experience Today}

If too much emphasis on current practice should not guide the design of a novel interface, it is crucial to understand the constraints and requirements that users have when they perform their work. It is important to understand why they cannot accomplish their work properly or perform it very well in a wide variety of situations. Both positive information and negative information on work practice are equally good to consider and analyze. User experience cannot be separated from the tools, methods and organizational setups that go with it. The difficult part is to access the right users, and not intermediary people who would synthesize user requirements for the design team. Obviously, all users cannot be accessed in all possible situations. However, by experience, selecting appropriate sets of users is much better than nothing! We need to remember that resulting acquired information is partial. This is why we need to have conceptual models that support interpretation and extrapolation in some cases. These conceptual models may be very loose and provided by domain experts in the form of narratives or simply active explanations of acquired information.

In our study, we have acquired such information from various flight attendants of five airlines distributed over the world. We chose these airlines because they were representative of various cultures, not only ethnical cultures but also corporate cultures. In addition, we tried to make sure that we had a variety of ages among the consulted populations. It was interesting to notice that with five airlines and roughly five flight attendants per airline, we converged toward very interesting categories of constraints and requirements. We used the Group Elicitation Method (GEM) to elicit their knowledge and knowhow. It worked perfectly once more time; GEM was used extensively and successfully in industrial settings for the last two decades [1]. The difficulty was not user-experience gathering itself. The difficulty was in the organization of the GEM sessions. Why? 
First, it is mandatory to carefully prepare with the actors of the various organizations to deal with such a participatory approach, i.e., users are not necessarily prepared to participate in a design team. Once they understood our goals and the way GEM sessions work, everything went very smoothly. In addition, it worked because GEM sessions were very carefully prepared in advance. Such a session typically starts with the right question asked to the selected set of users. In fact the right question can be split into several sub-questions, and we have a set of preliminary generic questions that are used to shape and develop the final appropriate question with domain experts. Here are some of these generic questions. What is the goal of the system that we plan to analyze, design or evaluate? How is the system or its equivalent being used (current practice, observed human errors)? How would you use this system (users' requirements)? What do you expect will happen if the corresponding design is implemented (e.g., productivity, aesthetics, and safety)? How about doing the work this way (naive and/or provocative suggestions)? What constraints do you foresee (pragmatic investigation of the work environment)?

Second, the social relation between the GEM facilitator and the selected users need to be extremely professional, i.e., the facilitator needs to be an ethnographer showing the GEM participants that what they are providing is clearly understood and will be effectively used. In addition, since such information is mostly made of stories, it must be kept confidential. The difficulty is in the interpretation and simplification of these stories.

In order to cross-fertilize data gathered from expert users, it is important to develop a cognitive walkthrough $(\mathrm{CW})$ that consists in exploring the user interface being designed, like a user would, to gather usability problems $[11,6]$. Before running a $\mathrm{CW}$ session, user scenarios and associated actions have to be created. Based on the scenario the evaluators explore the interface by asking appropriate questions for each action. Spencer (2000) suggested questions such as: "Will the user know what to do at this step?" or "If the user does the right thing, will he/she know that he/she did the right thing, and is making progress towards he/she goal?" We implemented this approach.

It is interesting to notice that results were very impressive. Two main categories emerge: (1) clear user requirements and constraints; and (2) open questions that remain unexplained. Both types were checked with experts. Some open questions were answered; others needed further investigations with an extended set of domain experts. In three months, we managed to have a set of validated requirements and constraints that were never found in the related industrial community. These results were used to specify a prototype.

\section{User Experience Tomorrow}

It is possible to analyze some parts of possible future user experience from experts, but there are situations, configurations or initiatives that will never be possible to anticipate and therefore only a prototype-based approach will enable the elicitation of possible use patterns. First, there are user behaviors that are standard and could be anticipated because they are related to a style of interface. The more the interface conforms a standard, the more user behavior will be predictable. Standardization is 
therefore a great incentive for future user experience prediction. Nevertheless, when new kinds of interfaces are designed and developed, usability predictability is no longer possible without an experimental protocol that involves a set of users facing a prototype.

Prototyping is then a key requirement in participatory human-centered design. Prototypes range from low fidelity, e.g., paper and pencil mockups, to high fidelity systems, e.g., carefully implemented realistic user interfaces. Interaction analysis has to be performed at different stages of the design and development process. During the early stages, methods such as GOMS [3] can be used to rationalize various interaction envelopes. Such analyses provide a good insight of the points that need to be further investigated and the ones that are obviously right or wrong. In our harmonization study, a first prototype was developed by a third party and it became obvious very soon that some parts of the interface should be redesigned using very simple GOMS analyses. We actually used the CogTool [4]. Human-centered design is inherently iterative, but budget requirements impose that the number of steps be minimal. Without using appropriate expertise, such minimization of the number of steps is impossible for a successful result in the end. For that matter, it is often crucial to spend some more time and money on a human-centered approach during the early stages of the design and development process to gain a mature product in the end. In other words, the more we could start with appropriate and relevant high-level user requirements and constraints, the best in the end, i.e., the more mature the product.

Interaction analysis needs to be performed constantly during the design and development process. It is like a Chef cooking a memorable dish. Experience and expertise play a great role, but incremental testing is a must. We do not insist enough on this necessary capacity of domain experts to be involved and concentrated during the design process. We often talk about latent human errors [9] that are committed during the design and development process and re-appear at use time, sometimes viciously. If we take a positive approach of this problem, latent errors deal with product maturity. Product maturity is a matter of constant testing. In an ideal world, we would have to test the product and its former prototypes in all situations in order to make sure that it will be fully mature at delivery time. This is obviously impossible, but designers and engineers must remember that the more situations they will experience in the use of the product, the better. This is a practice that happens to disappear with our current industrial way of managing projects. Indeed, engineers need to fill in spreadsheets and report all the time instead of fully concentrating on their design and development tasks. It seems that reporting has become more important than actual design and development! Motivation must be kept and creativity must remain the main asset of human-centered design teams. For that matter, reporting could be used in a different way that would effectively and significantly improve design. At this stage, it is important to make a distinction between reporting for work FTE (full-time equivalent) justification and writing for improving design. Writing for design will be discussed later in this paper.

Once analytical methods such as GOMS have been used and have produced their own results, prototypes must be used to assess remaining emerging user interaction issues. We then proposed new design features based on the integration of the following aspects: usability (e.g., legibility); perception (e.g., "gestaltgesetze" / laws of design); and aesthetics (e.g., "joy of use"). Complex interfaces require logical and 
consistent definition and implementation of graphic design elements that convey design hierarchy and structure, make correlations visible, express conditions, and both clarify functions and promote workflow. Elements of design are often both structurally and functionally related. Design is in no way random; in our project, each part of the interface has been developed to fulfill a specific function. The following factors were taken into account: the use of rasters/grids, the distribution and the size of fields, the use of typography, the alignment of individual as well as multiple elements, groupings, relations, choice of colors, the size and shape of elements. Throughout this process, importance has been placed on the underlying aspect of functionality - for example, interaction elements should enable users to navigate into the system, edit, enter data and read information. The underlying structure/hierarchy should be straightforward and enable clear understanding of the correlation between elements, as well as appropriate and timely feedback.

Then, it is time to bring back the prototype to selected users in order to evaluate it. This is what we did in the cabin display harmonization study. We went back to see flight attendants of the five airlines and presented them the developed prototype. Each session included four to five flight attendants. The prototype was initially presented, i.e., a walkthrough was performed with them. They could become acquainted with the interface in a couple of hours. Three scenarios were presented. Flight attendants were required to develop these scenarios using the prototype. At this point, we would like to emphasize the importance of scenarios in participatory human-centered design. They must be simple enough to be implemented in a reasonable period of time, but they must include the necessary steps required to induce the emergence of user behaviors. Each session was recorded. The debriefing in the end was performed as a GEM session, i.e., all flight attendants performed a brainwriting and concepts were categorized and prioritized. Results showed that the various interaction issues discovered could not be anticipated during the analytical phase. They were used to redesign the interface.

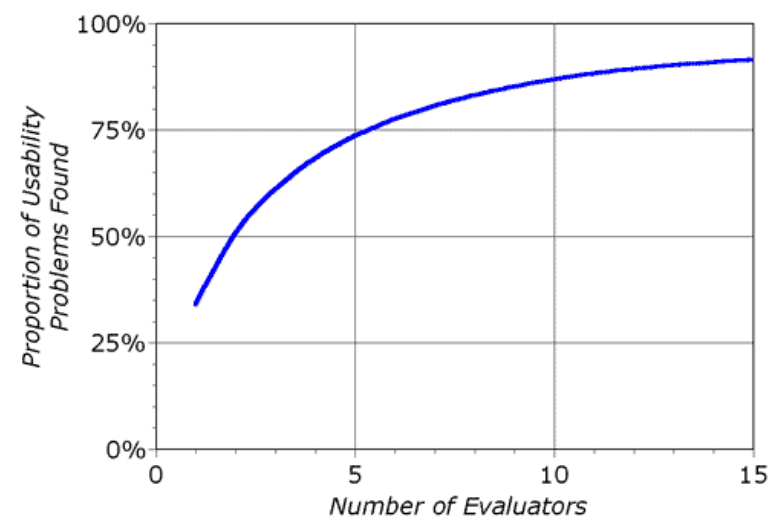

Fig. 1. Usability problems versus number of evaluators (Nielsen, 1994) 
It is interesting to confirm that Nielsen and Mack's findings [7] on the number of evaluators was in line with our GEM results concerning the evaluation of the prototype (Figure 1). Across five GEM sessions of five participants each (i.e., experts users), we converged toward very consistent results with an approximate variability factor of $25 \%$. More specifically, we elicited 24 human factors issues that were categorized into five main categories. These issues were very persistent, i.e., most human factors issue was elicited in more than 3 of 5 user groups (in our case, airlines) on 5. Some human factors issues were specific to one user group, and typically determined cultural differences.

\section{Identifying Where the Real Problems Are}

We already presented the -- today almost very classical -- way of how the participatory process could be carried out. However, it is as important to present where the real problems are in its implementation. First, we are talking about software engineering design. One of the most impressive attributes of software is its ease of modifiability. Since software is easy to modify, developers obviously do it with no reservation. This leads to a decrease of planning and forward thinking. The quality of high-level requirements stated in the beginning of the development process is crucial. If we do not start well, we are likely to modify the development trajectory all the time. Consequently, both product maturity and maturity of practice are directly impacted [2]. This is why user requirements and constraints are extremely useful in the definition of good high-level requirements for the product being designed. They enable to focus on practical and operational issues early enough in order to avoid later iterations on design flaws.

Industrial focus is often too much on technological solutions and not enough on what users really need to perform their work, i.e., users have to adapt to technology and not the other way around. However today, this is barely an engineering problem, it is a finance-driven management problem. Budgets are required to be reduced everywhere. But, the most important issue here is resource allocation. In fact, industry needs to hire more professionals, i.e., both human factors specialists and designers, to actually reduce their costs in the end. Such reductions are decided by finance-driven people and no longer by knowledgeable engineers. Therefore, participatory humancentered design (PHCD) budgets may look very unnecessary since they are not directly technology-centered, and immediately beneficial to the company. In addition, since PHCD has a major impact on maturity that is only observable at delivery time, finance-driven decision-makers do not see its relevance because this approach does not show immediate results, i.e., such a maturity-based approach is often perceived as a too long-term process. For that matter, this technique should be taught to top managers to get them more sensitive to what their company could gain in the end, and more importantly not loose time, money and employees' motivation by redesigning and repairing design flaws discovered at use time. Sometimes, it may happen that products are simply not acceptable to users and therefore rejected. Conversely, in our cabin display project, we clearly noticed that end users were not only pleased to be involved, but were potential champions of the PHCD approach and therefore resulting product. 
Despite the expertise of designers and human factors specialists, when you design a new human-machine system, there is always a part of unknown that generate many kinds of problems. First, decision-makers who fund the innovation project do not know exactly what to expect and may only be aware of partial judgments on an ongoing design process that may be related to the social relations between actors participating in the project. When there are external actors, such as airlines in our project, problems may arise from sensitive issues that may not be related to the design itself and decisions could be made not to go in a direction despite its user-centeredness. As a generic rule, participatory human-centered design, which involve a large number of actors related by commercial and legal issues, is only possible within socially acceptable boundaries. Awareness of these boundaries is often difficult to get at all relevant levels of the design chain. Second, developing appropriate scenarios is crucial, but we never know what would be the best generic scenarios to choose without a careful and knowledgeable user-centered analysis. This is why working with expert users is crucial. However, when these expert users are difficult to access, appropriate and efficient techniques are necessary to gather their knowledge and knowhow that will lead to the development of such human-centered design scenarios. We can confirm that GEM is a great technique to this end. It has been used extensively during the project and helped us a lot in the development of scenarios by providing episodes, cases, incidents, critical issues and relations between users and technology. It was interesting to notice that what we thought important in the beginning of the project happened to be a wrong choice, i.e., developing a very general interface that enables to process very complex cases. End users agreed on simplicity and it turned out that only a very simple set of interface objects were necessary to perform the tasks that they had to perform. Again, this requirement was given by previous inputs from people who were not in the real business of operating the kind of interfaces that we were harmonizing. Therefore, it is crucial to choose the right end users.

\section{Discussion}

From a general standpoint, it is very important and necessary to implement several complementary techniques to cross-fertilize design concepts and converge toward a harmonized user interface. First, analytical methods are important to determine an investigation envelope, i.e., the domain of harmonization. However, we need to be aware that this acquired knowledge is full of holes. Second, it is also important and necessary to involve real end-users, flight attendants in our study, to both fill in the holes left from the previous analytical step and determine additional issues. Third, there is no participatory human-centered design without the development of a prototype. This prototype should be developed with an integrated PHCD team, and not decentralized too long, e.g., in a software development company. Prototype design mainly consists in finding out all design elements (i.e., the ontology of the user interface made of fonts, shapes, colors and so on) and various functional aspects (i.e., consistency, structure, perception and so on). As already mentioned, the prototype incrementally evolves from low fidelity productions to higher fidelity productions. 
This evolution is the product of discussions with human factors specialists and inputs from user experts. It is crucial to carefully write design intentions and formative evaluation results to figure out where difficulties are: "Writing as design and design as writing" [8]. When you have difficulty to describe a concept, this is usually because this concept is not yet mature enough. Consequently, more work is required to improve the concept and return to the "drawing board" until you are able to "write" it correctly.

In the past, while developing human machine interfaces (HMI), designers, as well as human factors specialists, were not often involved in the specific teams because of technical constraints. Today, awareness and use of PHCD grows in industry. Humancomputer interfaces should be seen as mediating entities that transfer information back and forth between a system and a user - it is an important part of a communication process. This is why several university programs in design focus their curriculum on "communication design" or "information design" and concentrate exactly on these topics. Students learn to include both aesthetic and functionality perspectives in their work. Therefore, design decisions are not made arbitrary but rationally. What our project brought is a combination of backgrounds including human factors, design and engineering. The job of an information-designer is editing data in a graphical way that depends on the content. We always keep in mind that the product (digital or analog interfaces) should support the user to understand meaningfully all concepts involved in the interaction. In the past, interfaces were often typography-based, i.e., to get information the user had to read the text. Today, with the specific development and definition of design-rules that support the use of color, shapes, fonts, workflows (design-language), it is possible to show existing hierarchies, properties and links.

The more complex structures, information and connections are, the more necessary the participation of a designer in the development-team is. The job of a designer, in the sense of PHCD, is not only creating the surface, it is rather to run through all parts of the developing process of a project with the inclusion of all the influencing individual aspects. For example, the definition of the problem and a detailed analysis of all considered facts are mostly the first steps in the beginning. Skills such as conceptual, systematic and analytic working methods, abstract thinking and schematic description abilities are essential to design teams to succeed with complex topics. Working in interdisciplinary teams is important for the more and more complex tasks of recent projects.

Finally, we would like to insist on two main assets that a design team should have. First, design knowledge and knowhow is mandatory to start with the right approach on consistency and rational thinking, but also to make sure that a concrete mockup and later-on a prototype could support interactions among the members of the design team. Discussions are always better supported by a common frame of reference that should be as explicit as possible. This is also true when end users are involved in the participatory design process. Second, the expertise of the cognitive engineer who will be acquiring information from end users is crucial. The cognitive engineer of course masters knowledge elicitation techniques being used. However, his/her expertise should include knowledge of the domain, e.g., aeronautics in our case, and also knowhow of how to interact and socialize with end users. 


\section{References}

1. Boy, G.A.: The Group Elicitation Method for Participatory Design and Usability Testing. In: Proc. CHI 1996, the ACM Conference on Human Factors in Computing Systems, Vancouver, British Columbia, Canada, pp. 87-88 (1996)

2. Boy, G.A.: Knowledge management for product maturity. In: Proceedings of the International Conference on Knowledge Capture (K-Cap 2005), Banff, Canada. ACM Press, New York (2005)

3. Card, S.K., Moran, T.P., Newell, A.: The Psychology of Human-Computer Interaction. Lawrence Erlbaum Associates, Hillsdale (1983)

4. John, B.E.: The CogTool user guide. Carnegie Mellon University, Pittsburg, PA, USA (2008), http://www.cs.cmu.edu/ bej/cogtool/CogToolUserGuide-1_ $0 \_2 . p d f$

5. MoD HFI DTC. Cost arguments and evidence for human factors integration. Booklet produced by Systems Engineering \& Assessment Ltd., UK (2006)

6. Nielsen, J.: Usability Engineering. Academic Press, Boston (1993)

7. Nielsen, J., Mack, R.L.: Usability Inspection Methods. John Wiley \& Sons, NY (1994)

8. Norman, D.A.: Turn signals are the facial expressions of automobiles. Addisson-Wesley, Reading (1992)

9. Reason, J.: Human Error. Cambridge University Press, Cambridge (1990)

10. Spencer, R.: The Streamlined Cognitive Walkthrough Method, Working Around Social Constraints Encountered in a Software-Development Company. In: The Proceedings of CHI 2000, pp. 353-359 (2000)

11. Wharton, C., Bradford, J., Jeffries, J., Franzke, M.: Applying Cognitive Walkthroughs to more Complex User Interfaces: Experiences, Issues and Recommendations. In: The Proceedings of CHI 1992, pp. 381-388 (1992) 